Physica C 235-240 (1994)709-710

\title{
Layered Ba-K-Pb-Bi-O superconductor family: characterization of laser-ablated films
}

\author{
Marina Licheron ${ }^{\mathrm{a}}$, Isabelle Reynaud ${ }^{\mathrm{a}}$, Ricardo P.S.M. Lobo ${ }^{\mathrm{a}}$, François Gervais ${ }^{\mathrm{a}}$, Corinne Champeaux $^{\mathrm{b}}$, Pascal \\ Marchet $^{\mathrm{b}}$ and Alain Catherinot ${ }^{\mathrm{b}}$ \\ ${ }^{a}$ Centre de Recherches sur la Physique des Hautes Temperatures, \\ CNRS, 45071 Orléans Cedex 2, France \\ ${ }^{\mathrm{b}}$ Laboratoire de Matériaux Céramiques et Traitements de Surface, URA CNRS N 320 , \\ 87060 Limoges Cedex, France
}

\begin{abstract}
A layered structure of $\mathrm{Ba}-\mathrm{K}-\mathrm{Pb}-\mathrm{Bi}-\mathrm{O}$ systems presents superconductivity at critical temperatures twice higher than cubic structures of the same family. Increasing $\mathrm{Bi}$ concentration also increases $\mathrm{T}_{\mathrm{c}}$ but, on the other hand, prevents formation of the layered structure and kills superconductivity. It seems that this $2 \mathrm{D}$ structure can be forced by means of laser ablation processes. We present the first infrared reflection analysis of $\mathrm{Ba}_{2} \mathrm{BiO}_{4}$ laser ablated films and discuss their possible structure.
\end{abstract}

\section{INTRODUCTION}

A very interesting feature in the $\mathrm{Ba}-\mathrm{K}-\mathrm{Pb}-\mathrm{Bi}-\mathrm{O}$ superconductor family is the presence of superconductivity in both $2 \mathrm{D}\left[(\mathrm{Ba}, \mathrm{K})_{2}(\mathrm{~Pb}, \mathrm{Bi}) \mathrm{O}_{4}\right][1]$ and $3 \mathrm{D}\left[(\mathrm{Ba}, \mathrm{K})(\mathrm{Pb}, \mathrm{Bi}) \mathrm{O}_{3}\right]$ structures $[2,3]$. A property which is not present in any other system. Moreover, superconductivity is found to be twice higher in $2 \mathrm{D}$ structures, comparing with $3 \mathrm{D}$ ones. The main problem related to the production of $2 \mathrm{D}$ compounds is the incorporation of large $\mathrm{Bi}$ concentrations. More than $15 \%$ of $\mathrm{Bi}$ leads to the formation of a insulator double-perovskite structure. Unfortunately, rising bismuth contents in the $2 \mathrm{D}$ structure increases the critical temperature. Based on the 3D structure $(\mathrm{Ba}, \mathrm{K}) \mathrm{BiO}_{3}$ data, one could expect the highest $\mathrm{T}_{\mathrm{c}}$ for the layered structure in $(\mathrm{Ba}, \mathrm{K})_{2} \mathrm{BiO}_{4}$.

It seems that one of the best process in the production of superconducting films is the laser ablation. Excellent results have already been reported for $\mathrm{YBaCuO}$ with films presenting a high degree of orientation, high critical currents and sharp transitions [4].

In this work, we have tried to force the tetragonal (2D) structure in compounds with high Bi concentration with laser ablation. We present the first results of infrared characterisation of $\mathrm{Ba}_{2} \mathrm{BiO}_{4}$ films. A first analysis show that for smaller thickness, our films have the 2D structure but for thicker films it seems that a coexistence of 2D and $3 \mathrm{D}$ phases is developed.

\section{EXPERIMENTAL}

Films have been deposited using pulsed laser ablation. As a source, we have used an excimer laser operating at $248 \mathrm{~nm}(\mathrm{KrF})$ firing on sintered $\mathrm{Ba}_{2} \mathrm{BiO}_{4}$ pellets. In these pellets, we have only the double perovskite structure. In order to force the growth of the tetragonal structure rather than the cubic one we have used as substrate a $\mathrm{MgO}$ single crystal. The unit cell parameter of $\mathrm{MgO}$ is smaller than the one observed for the double perovskite and close to the unit cell parameter of the tetragonal phase. Anyway as the laser ablation process supposes that bonds between atoms are broken we can expect that the structure observed in targets will not necessarily be transposed to the films. Thus we shall expect that arriving at the substrate, the atoms will be disposed more favourably in the tetragonal layered phase.

Infrared reflection characterisation using a new Bruker IFS 307 spectrometer composed of two complementary interferometers covering a spectral range from far infrared up to ultraviolet. For our films we have measured room temperature reflection between 40 and $5000 \mathrm{~cm}^{-1}$.

\section{RESULTS AND DISCUSSION}

We show in Fig. I typical infrared reflection spectra obtained for such films. Film thickness has been determined from interference fringes in the near infrared region. For thicker films we can see that the global level of reflection is lower than for thinner ones in the region of $400-800 \mathrm{~cm}^{-1}$. Within this region lies a very strong reflection band of the $\mathrm{MgO}$ substrate. Around $500 \mathrm{~cm}^{-1}$ we find characteristic bands of the $a b$ plane of the tetragonal phase in $(\mathrm{Ba} . \mathrm{K})_{2}(\mathrm{~Pb} . \mathrm{Bi}) \mathrm{O}_{4}$ system. Thus the decrease of the reflection level at this region is just an effect of screening of the $\mathrm{MgO}$ band by the excitations observed in the film The thicker the film the more masked the $\mathrm{MgO}$ band is. The spectra can be quantitatively analysed in terms of a tour parameters oscillator model associated with simple interference calculations. The four-parameter model for the dielectric function $(\varepsilon)$, based on transverse (TO) and longitudinal (LO) optical frequencies $(\Omega)$ ) and their respective dampings $(\gamma)$ can be written as [5] 


$$
\frac{\varepsilon}{\varepsilon_{\alpha}}=\prod_{j} \frac{\Omega_{\mathrm{LOj}}^{2}-\omega^{2}+\mathrm{i} \gamma_{\mathrm{LOj}}^{\omega}}{\Omega_{\mathrm{TOj}}^{2}-\omega^{2}+\mathrm{i} \gamma_{\mathrm{TOj}}^{\omega}}
$$

Using Eq. 1 and the measured optical parameters of the $\mathrm{MgO}$ substrate we have calculated the oscillator parameters for the film through interference of normal incident light on the system film-substrate. Such a data analysis yields good fits for thinner films but does not fit well thicker ones. For the films which we have been able to determine the oscillator parameters we have found frequencies compatible with the ones present in the $a b$ plane of tetragonal $\mathrm{Ba}_{2} \mathrm{PbO}$. A clue to understand why thicker films cannot be fitted using the above model is given by preliminary measurements of X-ray scattering. It seems that for the first layers of deposited film we do force the tetragonal structure. By the way, increasing too much the number of layers (and thus film thickness) the cubic double perovskite phase starts developing. This means that thick films show a mixed structure: for the first layers in contact with $\mathrm{MgO}$ we find the 2D structure and as get away from the substrate the more stable cubic 3D phase is formed. Recent results have shown that this layered structured can also be formed in films with Potassium and Lead doping.

\section{CONCLUSION}

Infrared reflection measurements have been performed on laser ablated films of $\mathrm{Ba}_{2} \mathrm{BiO}_{4}$. A comparison of infrared data obtained for films and for tetragonal $\mathrm{Ba}_{2} \mathrm{PbO}_{4}$ shows that for thin films we have succeeded in forcing the formation of the tetragonal $\mathrm{Ba}_{2} \mathrm{BiO}_{4}$ by laser ablation methods. On the other hand, completing refiectivity data with X-ray diffraction measurements have allowed us to conclude that the tetragonal phase is not stable for thicker films.

\section{Acknowledgments}

RPSML thanks Brazilian agency CNPq.

\section{REFERENCES}

1. M. Licheron and F. Gervais, Phys.Rev. B 478008 (1993).

2. A. W. Sleight, J. L. Gillson, P. E. Biersted, Solid State Commun. 17, 27 (1975).

3. L. F. Mattheiss and D. R. Hamann, Phys. Rev. Lettt. 60, 2681 (1988).

4. R. P. S. M. Lobo, F. Gervais, C. Champeaux, P. Marchet, A. Catherinot, submitted to Phys. Rev. B

5. F. Gervais, J. L. Servoin, A. Baratoff, J. G. Bednorz and G. Binnig, Phys. Rev. B 47, 8187 (1993).

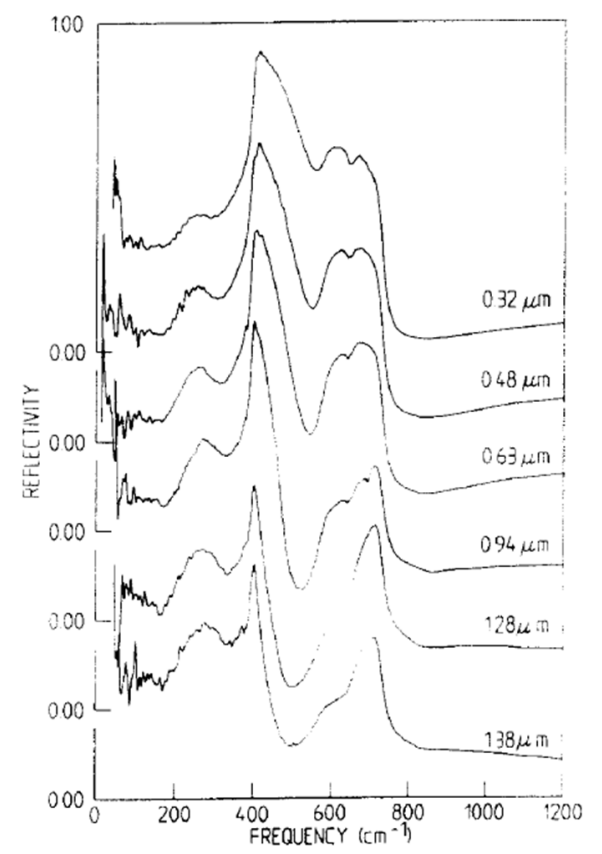

Fig. $1-\mathrm{Ba}_{2} \mathrm{BiO}_{4}$ film thickness dependence of infrared reflection spectra. 